Nuffield Department of Orthopaedics Rheumatology and Musculoskeletal Sciences, Botnar Research Centre, Oxford, UK

2 National Institute for Health Research Oxford Biomedical Research Centre Oxford, UK

Correspondence to: A Carr andrew.carr@ndorms.ox.ac.uk Cite this as: BMJ 2021;372:n339 http://dx.doi.org/10.1136/bmj.n339 Published: 09 February 2021

\section{Growing backlog of planned surgery due to covid-19}

\author{
Millions of people are now waiting and worried \\ Andrew Carr, ${ }^{1,2}$ James A Smith, ${ }^{1,2}$ Jenny Camaradou, ${ }^{1}$ Daniel Prieto-Alhambra ${ }^{1,2}$
}

Covid-19 continues to have a severe effect on planned surgery in the UK, and dealing with the resulting backlog is a critical concern for the NHS. Data from NHS England show that the number of patients awaiting treatment hit a record high of 4.46 million in November 2020, ${ }^{1}$ with the number of referrals well below 2019 levels. The same data suggest that roughly 2.3 million people are currently waiting for surgical care.

Though the number of surgical patients treated within the 18 week NHS standard is improving from its worst point in the first wave of the pandemic, the number who have already waited more than a year for treatment has reached 138 401, a 153-fold increase from 904 in November 2019, and growth shows no sign of slowing. Trauma and orthopaedics, oral surgery, and plastic surgery seem particularly affected, but even cancer surgery has been delayed: the percentage of patients having cancer surgery within one month of the decision to treat dropped from $92 \%$ to $88 \%$ over the same period. ${ }^{2}$

Why have waiting times increased so much? Solid evidence is not yet available, but several contributors are likely. Operating theatres and outpatient clinics were closed as they became needed to treat patients with covid-19 during the first wave in 2020. Surgical staff, particularly junior surgical and nursing staff, were redeployed to provide cover for extra beds occupied by patients with covid-19 and for staff unable to work because they had covid-19 or were isolating. Despite better availability of protective equipment, hospitals remain high risk areas for acquiring the infection. The procedures put in place to protect patients and staff mean that seeing and treating patients in hospital takes much longer than it did before the pandemic. Delays or failures in patient testing have also been problems at times, wasting much needed space on operating lists. Workforce shortages may be amplified by increased viral transmissibility in the current wave.

The NHS has enjoyed substantial public, political, and media support during the pandemic. However, the historical lack of spare capacity in the NHS has arguably resulted in poor resilience and weakened its ability to cope with a stressor such as covid-19. The full effects of limited capacity will become clearer only as the long term consequences of delayed care are better characterised. Although many patients have been understanding and are waiting patiently for the situation to improve, they have concerns about delays and the lack of information about expected new timelines.

Patients also want reassurance that they will be safe from covid-19 when they are admitted. Governments must develop communication strategies that identify patients' concerns and misconceptions about risk of infection and provide information that patients can trust. Individual differences in perception of risk are key to effective communication, as is open acknowledgment of uncertainty. Patients want information that is easy to find, transparent, consistent, timely, and understandable so that they can make more informed decisions about their treatment and contingency plans. While many patients embrace digital technologies, care must be taken to address the growing digital divide affecting hard to reach groups as well as other emerging health inequalities.

Predictive modelling suggests that around 28 million operations were cancelled or postponed globally during the peak 12 weeks of the first wave ${ }^{3}$; this number will surely increase as the pandemic progresses. The ability to clear the resulting backlog will depend on the resources available in different countries, and will be a serious challenge for many. In the US, one study estimated that a backlog of at least one million orthopaedic surgical cases would remain two years after elective surgery stopped being deferred because of covid-19. 4

As the huge task of clearing the backlogs begins, surgical teams must be provided with appropriate resources, facilities, and both professional and mental health support. ${ }^{6}$ One promising route to increasing the volume of surgical care in the UK is so called "green pathways"-covid-free areas of hospitals where planned surgery can continue with substantially reduced risk to patients and staff. New ways of working with remote consultations, community diagnostic hubs, increased use of the independent sector, and regional treatment hubs with ring fenced resources for planned care are already emerging in many areas. ${ }^{7}$ One concern, however, is that extra capacity in the independent sector is concentrated in the south east and not where it is most needed.

Some regions are increasing activity by pooling waiting lists, prioritising cases, and removing patients from waiting lists who no longer need surgery. Many patients have had to accept compromises, including reduced choice about where and by whom they are treated. Clear and regular communication with patients regarding local plans and the likely timescale for their surgery remains critical.

Additional resources and greater capacity will not be enough. Profound changes to the way we work will be also be required, along with reform to create a leaner, more cost effective, and more flexible NHS able to make nimble decisions in response to crises such as covid-19. 


\section{EDITORIALS}

Competing interests: We have read and understood BMJ policy on declaration of interests and declare JC is a lay member of the NICE covid-19 expert panel.

Provenance and peer review: Commissioned; not externally peer reviewed.

We thank our surgical colleagues Neil Mortenson, Jonathan Rees, Andrew Price, Matt Costa, Dan Perry, Anthony Palmer, David Beard, Jeremy Rodriguez, Abhilash Jain, Chris Lavy, Tim Theologis, and Amar

Rangan and patient representatives Carol Brennan and Dair Farrar-Hockley for input.

1 NHS England. Consultant-led referral to treatment waiting times data 2020-21.

https:/www.england.nhs.uk/statistics/statistical-work-areas/rtt-waiting-times/rtt-data-2020-21/

2 NHS England. Cancer waiting times. https://www.england.nhs.uk/statistics/statistical-work-areas/cancer-waiting-times/

3 COVIDSurg Collaborative. Elective surgery cancellations due to the COVID-19 pandemic: global predictive modelling to inform surgical recovery plans. Br J Surg2020;107:1440-9.pmid: 32395848

4 Jain A, Jain P, Aggarwal S. SARS-CoV-2 impact on elective orthopaedic surgery: implications for post-pandemic recovery. J Bone Joint Surg Am 2020;102:e68. doi: 10.2106/JBJS.20.00602 pmid: 32618916

5 Fowler AJ, Dobbs TD, Wan YI, etal. Resource requirements for reintroducing elective surgery during the COVID-19 pandemic: modelling study. Br J Surg 2021;108:97-103.

doi: 10.1093/bjs/znaa012

6 Royal College of Surgeons. Supporting the wellbeing of surgeons and surgical teams during COVID-19 and beyond. 2020. https://www.rcseng.ac.uk/coronavirus/recovery-of-surgical-services/tool-6/

7 Royal College of Surgeons. Managing elective surgery during the surges and continuing pressures of COVID-19. 2020. https://www.rcseng.ac.uk/coronavirus/recovery-of-surgical-services/tool-7/

This article is made freely available for use in accordance with BMJ's website terms and conditions for the duration of the covid-19 pandemic or until otherwise determined by BMJ. You may use, download and print the article for any lawful, non-commercial purpose (including text and data mining) provided that all copyright notices and trade marks are retained. 